\title{
Natural Media Workshop
}

\author{
Carl H. Smith \\ LTRC, Ravensbourne \\ London, UK \\ c.smith@rave.ac.uk
}

\author{
Robert Pepperell \\ FOVOLAB \\ Cardiff Metropolitan University \\ Cardiff, Wales, UK \\ rpepperell@cardiffmet.ac.uk
}

\author{
Jazz Rasool \\ LTRC, Ravensbourne \\ London, UK \\ j.rasool@rave.ac.uk
}

\author{
Tony Langford \\ FOVOLAB \\ Cardiff Metropolitan University \\ Cardiff, Wales, UK \\ tolangford@cardiffmet.ac.uk
}

\author{
Nick Lambert \\ Ravensbourne \\ London, UK \\ n.lambert@rave.ac.uk
}

\author{
Kim Veltman \\ Virtual Maastricht McLuhan \\ Institute \\ The Netherlands \\ kimveltman@hotmail.com
}

\section{OVERVIEW}

This workshop will examine what our current imaging and sensing technologies do to our perception. We will examine, using practical examples, the potential to develop more 'Natural Media' and technologies by broadening the focus of attention to the whole visual, auditory, tactile and sensual field. The aim is to re-incorporate peripheral awareness into our experience using these multiple sense inputs.

It is a common experience to register activity at the edges of our vision, our peripheral vision (to 'see out of the corner of one's eye') but this concept is not often discussed relative to our other senses. What exactly is peripheral hearing? What about peripheral touch, smell or taste? What would it be like to have access to the periphery of these senses?

Both 'immersive' and non-immersive technologies present visual information in an unnatural way, often forcing us to narrow our field of attention to the central area of vision, thus ignoring most of the peripheral field. How can technology help us to become more present and open to the breadth of our perceptual experience? Overly narrow, misdirected, or hyper-focused attention can lead to stress, anxiety, and other damaging physical and psychological conditions.

'Natural Media' aims to uncover what happens when we move from direct experience to living through photographs, paintings, films and VR. Linear perspective relegated the significance of peripheral vision and therefore the full contextual experience of what we see and can therefore know.
It is important to focus on more than one predominant sense (vision) in order to start realigning and potentially redesigning our perception using 'Natural Media'. Thus, this workshop will explore what is the peripheral space of the other senses such as hearing, touch, taste and smell and in addition explore artificial senses such as the recently released 'North Sense'.

Vision: Fovography is a method of recording the $3 \mathrm{D}$ world on a 2D surface more naturally than can be achieved using devices based on linear perspective, such as cameras and computer graphics engines. It is based on hundreds of years of experimental work by artists and architects on the best ways to depict human visual experience, and draws on novel research in vision science and psychology. We will give participants the opportunity to experience this perceptual technology in action. This draws on our successful research symposium at the British Computer Society in November 2016, where we partnered with Kinetica, the Computer Arts Society and Ravensbourne to discuss and demonstrate Fovography displays and content to a range of researchers form several different disciplines.

Our research into human visual perception has led to several technological developments that offer improved ways of depicting visual experience. Existing imaging systems, such as cameras and computer graphics engines, are based on the geometry of linear perspective, which was developed over 500 years ago during the Italian Renaissance. Useful as linear perspective has been in allowing us to capture visual space on a flat picture plane, its many limitations have been well known ever since it was discovered. These include 
the fact that perspective images generally capture only a single eye point of view, and a very narrow angle of vision.

Fovography (www.fovography.com) overcomes these limitations by allowing us to capture the full field of view (hence the prefix 'fov') and present it on a flat surface in a way that appears natural to human perception. Moreover, because we represent the human visual field in a natural way, the resulting images appear to have much more breadth and much more depth than conventional images. This means we can create, in effect, 3D visual experiences without glasses, goggles, or expensive screens (www.fovography.com).
Audio: The NERVANA headphone system electronically signals the Vagus nerve which in turn stimulates the release of neurotransmitters in the brain, which has the potential to entirely revolutionise how we experience sound. Also, the HEARNOW Here Active Listening system, allows users to raise or lower the volume of the world around them and remove certain sounds in one's environment.

Tactile: The SUBPAC tactile interface is a wearable 'Tactile Bass System' that transfers the physical dimension of sound directly to the body. Whether being used to enhance music or as a vehicle for accessing physical immersion in VR, $M R$ and gaming, the SubPac adds a powerful new sensory element to any audio experience. 